\title{
Detecting of Periodic Fasciculations of Avian Muscles Using Magnetic and Other Multimedia Devices
}

\author{
Isao Nakajima ${ }^{1 *}$, Sachie Tanaka ${ }^{2}$, Kokuryo Mitsuhashi $^{3}$, Jun-ichi Hata ${ }^{4}$, Tomo Nakajima ${ }^{5}$
}

\begin{abstract}
In the past, there was a theory that influenza wasn't transmitted directly from birds but was infected to humans via swains. Recently, molecular level research has progressed, and it was confirmed that the avian influenza virus can directly infected to human lung and intestinal epithelial cells. Three pandemics in the past 100 years were also infected to humans directly from birds. In view of such scientific background, we are developing a method for screening sick birds by monitoring the physiological characteristics of birds in a contactless manner with sensors. Here, the movement of respiratory muscles and abdominal muscles under autonomic innervation was monitored using a magnet and Hall sensor sewn on the thoracic wall, and other multimedia devices. This paper presents and discusses the results of experiments involving continuous periodic noise discovered during flight experiments with a data logger mounted on a Japanese pheasant from 2012 to 2015.

A brief summary is given as the below:

1. Magnet and Hall sensor sewn to the left and right chest walls, bipolar electrocardiograms between the thoracic walls, posterior thoracic air sac pressure, angular velocity sensors sewn on the back and hips, and optical reflection of LEDs (blue and green) from the skin of the hips allow observation of periodic vibrations(fasciculations) in the waves. No such analysis has been reported before.

2. These fasciculations are presumed to be derived from muscle to maintain and control air sac pressure.

3. Since each muscle fiber is spatially Gaussian distributed from the sympathetic nerve, the envelope is assumed to plot a Gaussian curve.

4. Since avian trunk muscles contract periodically at all time, we assume that the sympathetic nerve dominates in their control.

5. The technique of sewing a magnet to the thoracic wall and measuring the strength of the magnetic field with a Hall sensor can be applied to screen for early stage of avian influenza, with a sensor attached to the chicken enclosure.
\end{abstract}

Key words: Avian influenza, Autonomic nerve, Pheasant, Hall sensor, FFT/IFFT.

\section{BACKGROUND}

\subsection{Purpose of this study}

This paper presents and discusses continuous periodic muscle fasciculations observed in the avian trunk, a phenomenon not observed in mammals; and apparently attributable to the autonomic nerves, magnets and sensors monitoring multiple properties: potential, barometric pressure, acceleration, and optical reflex.

\subsection{Avian influenza}

The global poultry industry (e.g., chicken, quail, ducks) involves some 20 billion individual animals, with an estimated market size of $\$ 120$ billion per year. The type of the influenza $(\mathrm{H} 1, \mathrm{H} 2, \mathrm{H} 3)$ responsible for global pandemics have been demonstrated to be transmitted directly to humans from birds via the low pathogenic avian virus. No genetic traces indicating transmission through swine have been detected in the past three pandemics.

The 2009 WHO declaration of a pandemic due to swinederived viruses appears to have been significantly overestimated. The last novel influenza was of the swine type, and did not result in a pandemic, with mortality similar to that of seasonal influenza. The WHO Secretary General responsible for issuing the Pandemic Declaration was evacuated from the European Union. We need to pay close attention to the older concept s regarding flu policy [1]-[3].

Several reasons have been proposed to explain human infection from birds. Two explanations predominate:

Human sialic acid receptor: Sialic acid is present in vivo at the end of sugar chains on the surface of cell membranes as a component of complex carbohydrates with long hydrocarbon chains called ceramides [4].

Manuscript received December 09, 2019; Revised December 24, 2019; Accepted December 25, 2019. (ID No. JMIS-19M-12-053)

Corresponding Author (*): Isao Nakajima, Shimokasuya 143, Isehara City, Japan, +81-463-91-3130, jh1rnz@aol.com.

${ }^{1}$ Tokai University School of Medicine, Isehara City, Japan, jh1rnz@aol.com

${ }^{2}$ Dept. of Laboratory Animal Science, Support Center for Medical Research and Education, Tokai University. Isehara City, Japan. baron@is.icc.u-tokai.ac.jp

${ }^{3}$ Seisa University Oiso Campus, Oiso Town, Japan. k_mitsuhashi@ seisa.ac.jp

${ }^{4}$ Central Institute for Experimental Animals, Kawasaki City, Japan. hata@ciea.or.jp

${ }^{5}$ Okinawa Prefectural Yaeyama Hospital, Ishigaki City, Japan. T.Nakajima0225@outlook.com 
This acid has important biological functions. Of these, the sialic acid receptor targeted by the influenza virus in the early stages occurs most frequently in the respiratory system and digestive system. Since sialic acid is present at the tip of this complex carbohydrate, it is called a sialic acid receptor.

In fact, sialic acid receptors are found in abundance in $\alpha 2,3$ or avian receptors in the deep lung (respiratory bronchioles and alveolar cells); $\alpha 2,6$ is found in the upper respiratory tract. That is, there are mainly human receptors, especially in infants, and when infants are infected with H5N1, pneumonia becomes severe [5] (Figures 1 and 2).

While, the frequency of appearance is relatively modest, $\alpha$ 2,3 type ebbs and flows in small intestinal epithelial cells over time. Human genes encode for both $\alpha 2,3$ and $\alpha 2,6$. This means that the earlier hypothesis that only pigs and ferrets(weasel) have both types and that viruses cannot infect humans through these intermediate hosts have not been substantiated. Recent studies indicate that H7N9 can hybridize with lowpathogenic viruses and seasonal influenza viruses to achieve human infectivity.

Birds do not have a diaphragm, and the position and size of the avian heart and the rotation of the aorta (not the left) differ markedly from those of mammals. Furthermore, as avian heart rates are extremely fast $(200-400 / \mathrm{min})$, it is necessary to devise measures such as time constants and measures against low potential when gathering data related to bird electrocardiograms. Infection by highly pathogenic avian influenza disturbs both breathing and he autonomic nerves (sympathetic and parasympathetic nerves), generating arrhythmias in electrocardiograms and ultimately, it is presumed, leading to cardiac arrest. Given this backdrop, we believe it is essential to screen affected individuals and to perform bird respiration monitoring and autonomic nervous system monitoring using ICT devices.

Against this background, we have developed an esophageal catheter to analyze the physiological functions of birds using multimedia technology, and wireless packet communication devices that attached wild whooper swans to collect their respiratory rate and heart rate [6-16]. In the past, physiological studies on birds and the relationship between autonomic nerves and electrocardiograms in chickens have been reported [17]-[27].

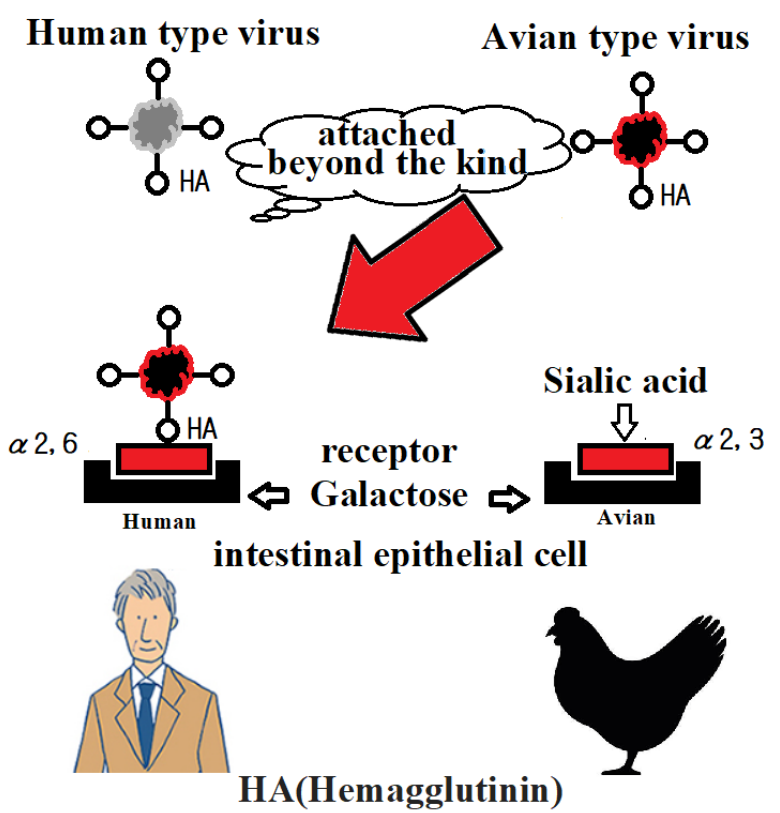

Fig. 1. While avian-type viruses are generally believed not to bind to human sialic acid receptors $(\alpha 2,6)$, c ounterexamples exist [4].

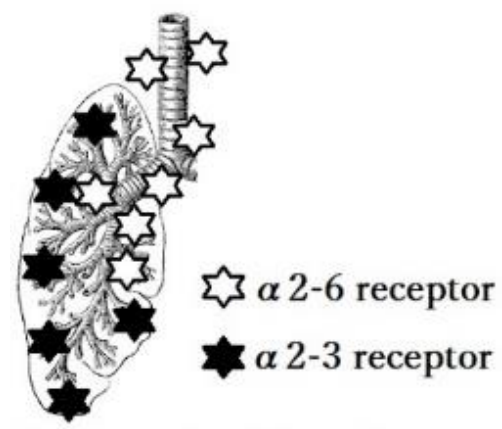

lung, tracheal, bronchus

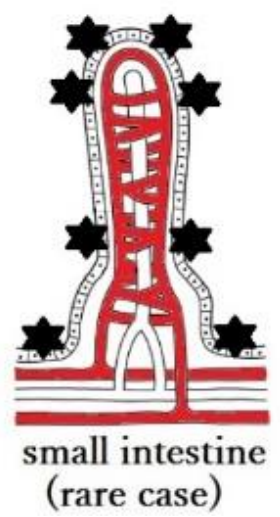

Fig. 2. Avian-type sialic acid receptors are present in the lungs and intestines of infants and the elderly, pr oviding a direct route for infection by the avian influ enza virus [5].

\section{METHODS}

\subsection{Signals Buried Noise}

Measurement uncertainty and noise can make it difficult to spot the oscillatory behavior of a signal, even if such behavior is expected. Several techniques are available for detecting periodic signals buried in noise. In this experiment, these five (electric potential, magnet, pressure, vibration, force) time-series-signals can be generalized as shown in the following equation: 


$$
\mathrm{Y}_{\mathrm{t}}=\mathbf{a}+\sum \mathrm{Ai} \mathrm{Y}_{\mathrm{t}-\mathrm{i}}+\mathrm{W}_{\mathrm{t}}
$$

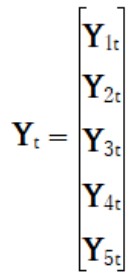

$\mathbf{W}_{\mathrm{t}}$ : Mean value $=0$, and follows a five-dimensional distribution of covariance matrix

This method calculates a statistic, making it possible to detect a signal of relatively strong amplitude. The drawback is that a signal with low amplitude will remain in the residual (Wt). We applied another approach, the classic frequency spectrum analysis method called FFT (Fast Fourier Transform). We selected a specific frequency band and reconstructed waveforms using IFFT (Inverse Fast Fourier Transform). Periodic signals detected in noise cannot be noise; additionally, if the signal is recognized in multiple dimensions and correlations (for example, in similar envelopes) are detected between the signals, an independent signal is likely to affect each dimension.

\subsection{Key Technologies (Magnets and Hall sensors)}

As discovered by Coulomb and summarized in Coulomb's law of electrostatic force, magnetic force acts between magnetic poles at magnitudes proportional to the product of the respective magnetic quantities $\mathrm{ml}$ [Wb] and $\mathrm{m} 2[\mathrm{~Wb}]$ and inversely proportional to the square of the distance $r[\mathrm{~m}]$ (Figure 3 ).

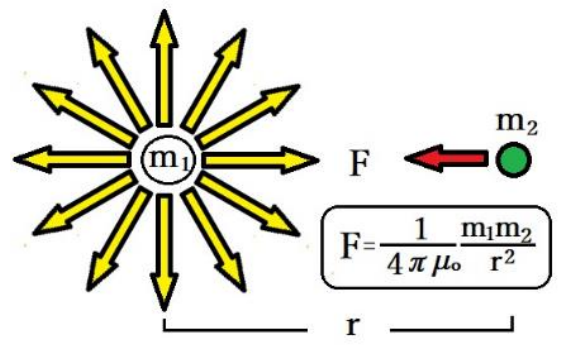

Figure 3 The force"Fig. 3. Force $F$ "' generated between two points is inversely proportional to the square of $t$ he distance" $r "$ ".

For p-type or n-type semiconductor samples, a current $I$ is passed in the $x$ direction and a magnetic field $B$ is applied in the $\mathrm{z}$ direction (Figure 4). Charged particles (carriers) flowing through the sample have Lorentz force $\mathrm{F}$ due to the magnetic field and are accelerated along the $\mathrm{y}$ axis.

As a result, carriers accumulate on the surface of the $\mathrm{s}$ ample, an electric field (Hall electric field) is generated in a direction orthogonal to both the current and the ma gnetic field, and an electromotive force Vac is generate d. The Hall effect can be applied to the detection of the magnetic field strength by the Hall element.

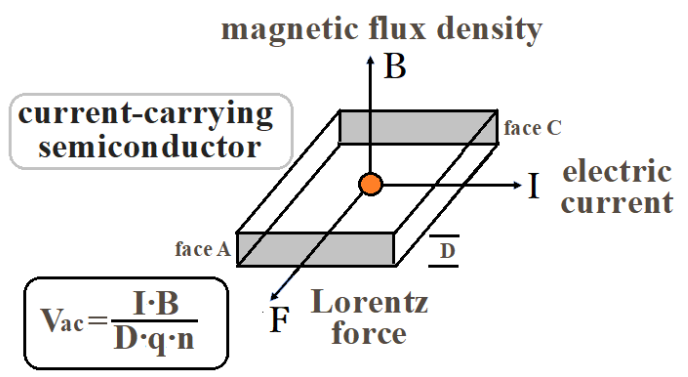

Fig. 4. $\mathrm{V}_{\mathrm{ac}}$ is generated in the semiconductor in th e environments of $\mathrm{B}, \mathrm{I}, \mathrm{D}$, and F.

As mentioned above, force $F$ is inversely proportional to the square of distance. If $\mathrm{m} 1$ and $\mathrm{m} 2$ are constant and the strength of the magnetic field changes, the distance has changed. In this experiment, changes in the magnetic field are measured using a semiconductor device called A132A, as the change in distance between two points is monitored. Figure 5 gives the circuit diagram.

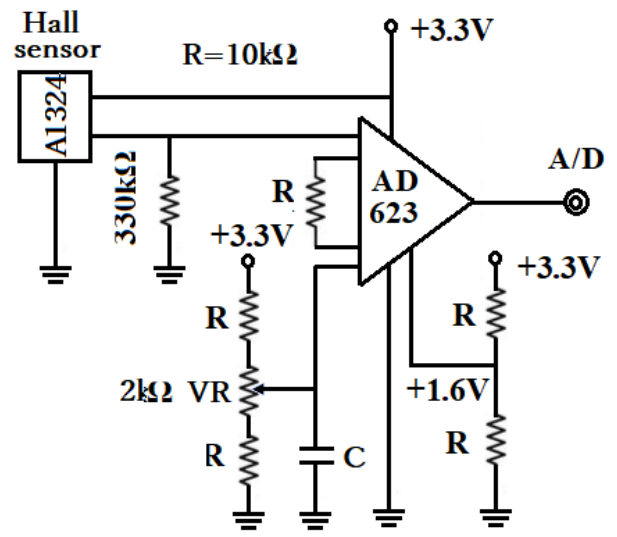

Fig. 5. Hall sensor circuit with OP AMP.

\subsection{Mathematical Analysis}

\section{- Fast Fourier Transform (FFT)}

If $\mathrm{x}(\mathrm{t})$ is a time signal and $\mathrm{X}(\mathrm{f})$ its frequency component, the Fourier series is expressed as the sum of the fundamental wave $\mathrm{fO}$ and its integral frequency component. Applied here is a fast Fourier transform using butterfly computations, a division procedure. With FFT, time axis data for a certain time (frame) is cut out and calculated. The number of samples per frame must be a power of 2 . Here, 1024 is one frame.

\section{- IFFT (inverse Fast Fourier Transform)}

Sampling at 1,000 [Hz], a carrier frequency of $50-60 \mathrm{~Hz}$ amplitude, and an envelope (about 5-30 Hz) amplitudemodulated for that signal are used to resolve a specific frequency (e.g., $60 \mathrm{~Hz}$ for ECG reconstructed with IFFT or over $20 \mathrm{~Hz}$ for light reflections, depending on the site). This research seeks to confirm the source of this carrier signal. 
What does the source of this carrier signal and the meaning of the envelope?

\subsection{Data logger}

Physiological analyses of birds require signal processing technologies. We developed an ultra-compact data logger that birds are capable of carrying without impeding flight. Table 1 gives the specifications; Figure 6 shows a conceptual diagram; Figure 7 presents a block diagram.

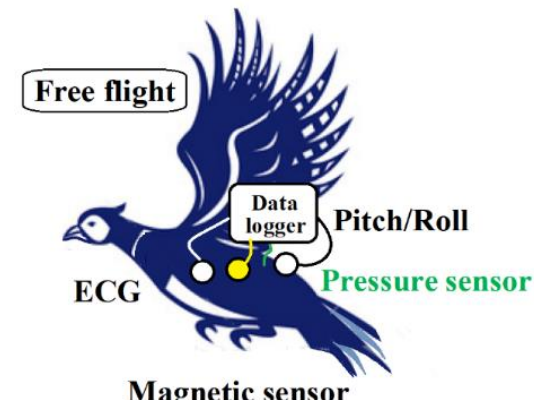

Fig. 6. Conceptual diagram of the measurement.

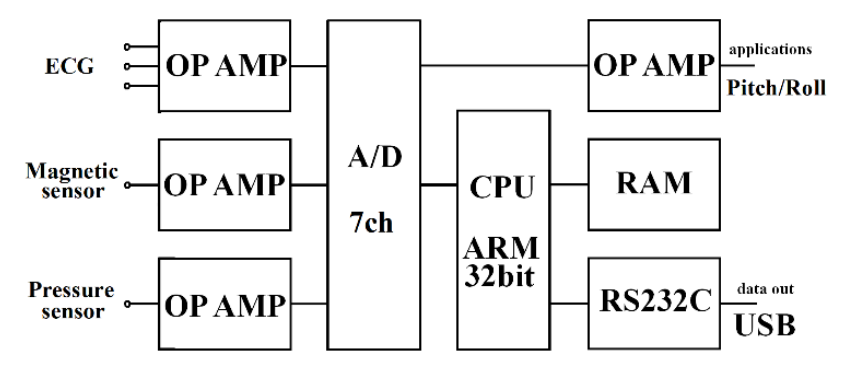

Fig. 7. Block diagram for data logger.

Table 1. Data logger specifications.

\begin{tabular}{|l|l|}
\hline CPU & ARM 32 bit \\
\hline Battery & $3.7 \mathrm{~V}$ \\
\hline A/D conv. & A / D 14 bits, Max $1000 /$ sec. 7 ch.s \\
\hline Memory & $100 \mathrm{MB}$ \\
\hline Data Output & Connected to the PC via USB \\
\hline Total weight & $32 \mathrm{~g}$ \\
\hline
\end{tabular}

\subsection{Sensing devices}

The Hall sensor for magnetic field measurements and other devices were sewn to the pheasant as follows:

\section{A: Magnetic field}

As shown in Figure 8, the magnet and Hall sensor were sewn with silk thread on the left and right thoracic walls (sixth intercostal space).

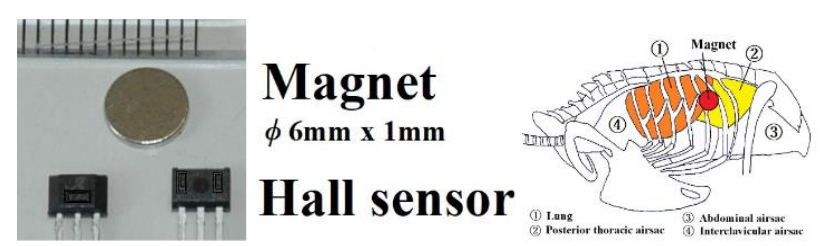

Figure 8 Magnet and Hall sensor, sensing position at thoracic wall
A bipolar electrocardiogram electrode (silver electrode, $8 \mathrm{~mm}$ diameter) was sewn subcutaneously on the head side (left and right chest walls) where the magnet was sewn. The neutral point was positioned under the midline of the pelvis.

\section{C: Barometric pressure (posterior thoracic air sa c pressure, oral cavity pressure)}

A drain was inserted into the posterior chest air sac with a vinyl tube with an outer diameter of $2 \mathrm{~mm}$. This was secured with silk thread, after which an atmospheric pressure sensor was connected. For intraoral pressure, a 50\% area was partitioned in a $10 \mathrm{~mm}$ diameter cylinder. Air pressure was measured according to Bernoulli's theorem.

\section{D: Vibration (thoracic wall, pelvis)}

One angular velocity sensor (Murata ENC-03R) is based on the principle whereby the Coriolis force affects the rotational angular velocity applied to the vibrating body. This element was sewn with silk thread along the midline of the pelvis or midline of the back. The two are orthogonal and capable of detecting vibrations indicating pitch axis and roll axis.

\section{E: Optical reflected power}

Two LEDs (blue and green) covered with a black shield illuminate the side of the pelvis to create a structure that blocks direct light. Light reflected from the skin is received by an optical sensor and amplified. This is the same principle applied to detect $\mathrm{SaO} 2$. This optical reflection is considered to be transmitted through the skin and reflected from muscles.

\section{EXPERIMENTS}

\subsection{Target animals}

- Female pheasants: 26; average weight 740 g

- Experiment place: Outdoor, tennis court (Oiso Campus, Seisa University)

Japanese pheasant females were chosen for the study because they are bred as poultry, readily available, and feature developed skeletal muscles, allowing relatively easy measurement of muscle signals. Figure 9 illustrates aspects of the flight experiment.

\section{B: ECG}




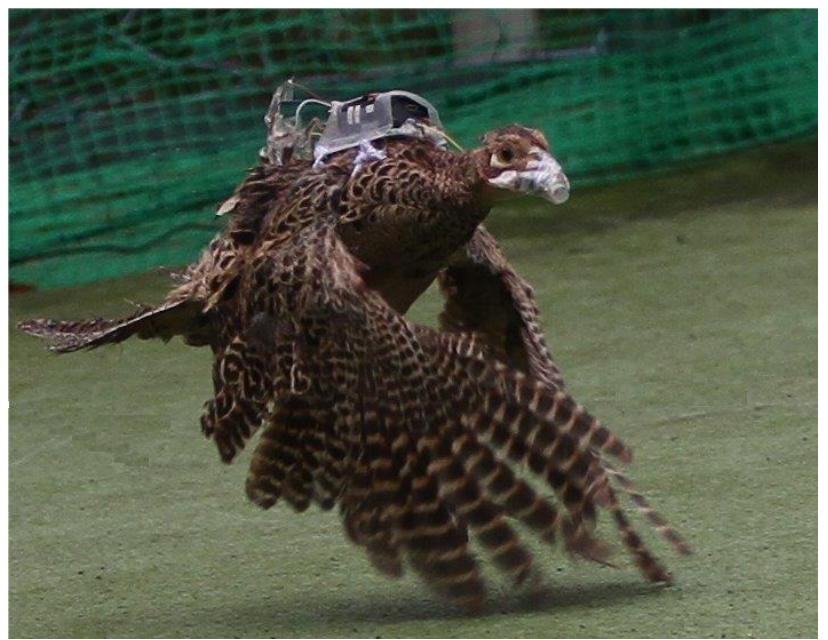

Fig. 9. Experiment involving flying pheasant.

\subsection{Experimental Results}

The results of the flight experiment are as follows. Periodic noise is observed for all pheasants at different frequencies at rest (in this case, before flight) and after flight.
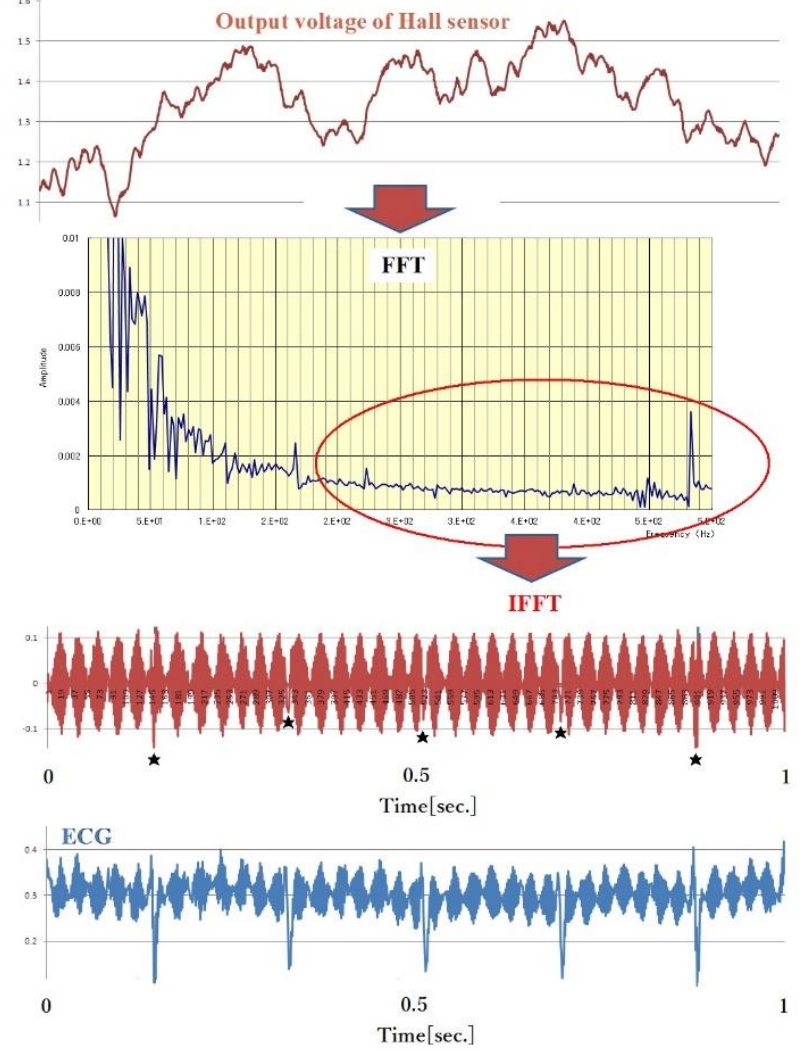

Fig. 10. Magnetic waves at top; IFFT wave is shown at th e $3^{\text {rd }}$ stage; the $4^{\text {th }}$ stage is the ECG at the same thoracic wall.

\section{A: Magnetic field}

Figure 10 shows the change in magnetic field strength detected by the Hall sensor. If we convert these signals by FFT and IFFT at high frequencies (higher frequency), they become third stage periodic noise. The lower area of Figure 10 shows an electrocardiogram recorded at the same site and at the same time. Both contain periodic noise that synchronizes, possibly due to the vibration of the intercostal muscles of the thoracic wall oscillating at a distance, which is riding as an amplitude modulation like AM radio signals. This phenomenon could be due to the intercostal muscle's mechanical vibration(fasciculations).

\section{B: ECG}

As shown in Figure 11, a periodic ECG electrode placed on the chest wall detects periodic noise, assumed to come from intercostal or other muscle's electrical vibration (fasciculations).

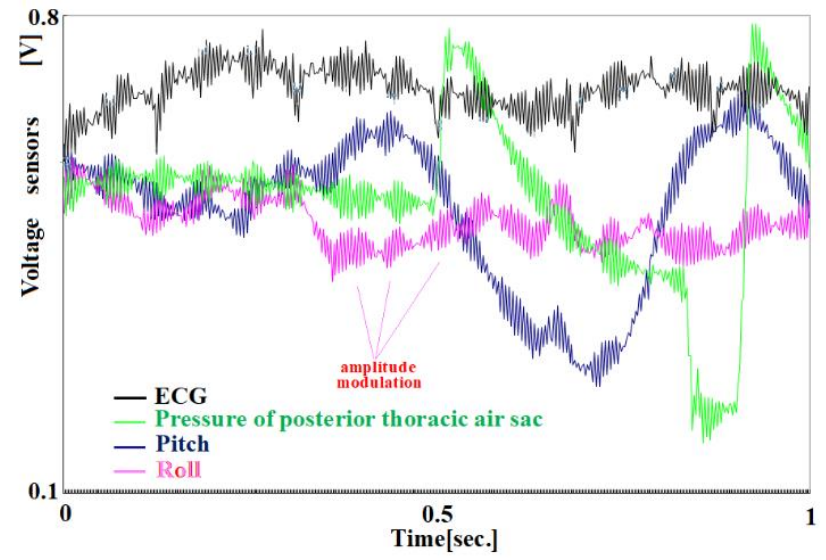

Fig. 11. Correlation between ECG, posterior thoracic air sac pressure, and angular velocity (pitch, roll) after flight.

\section{C: Pressure}

As shown in Figure 11, the pressure of the posterior thoracic air sac has a periodic signal that is amplitudemodulated by its pressure. This is believed to be due to the external tightening force generated by the upper pelvis muscle's vibration (fasciculations).

\section{D: Angular velocity}

As shown in Figure 11, vibrations affect both the pitch angle and the roll angle sewn on the chest wall and are synchronized with electrocardiogram noise. This vibration is believed to originate from intercostal muscles.

\section{E: Optical reflection}

The light energy reflected by the skin (the side of the hindquarters) from the LED indicates periodic vibrations in both green and blue. The muscles on the side of the pelvis and the oblique muscles have a significant role in these vibrations(fasciculations).

Table 2 Frequency components and amplitude components before and after the flight compared with power spectrum analysis 


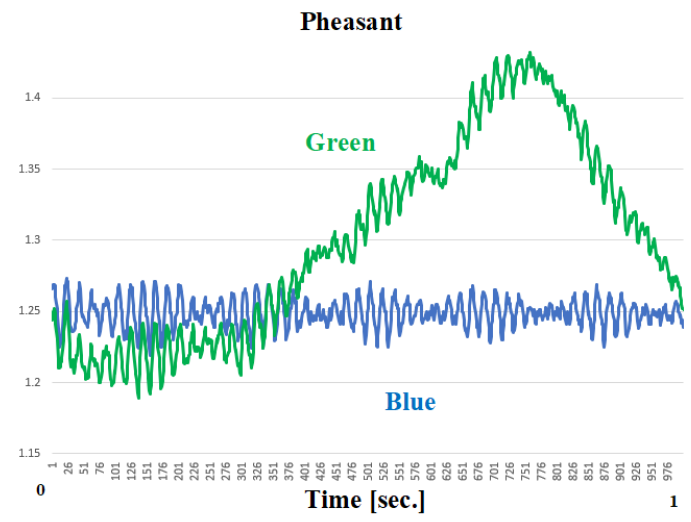

Fig. 12. The light energy reflected by the skin from the LED shows periodic vibrations in both green and blue.

\subsection{Changes before and after flight}

The frequency components and amplitude components of the envelope before and after the flight were compared with power spectrum analysis(Figure 11). Table 2 presents the amplitude of the peak of envelope before and after flight.

Table 2. Peak of envelope before and after flight.

\begin{tabular}{|c|c|c|c|c|}
\hline \multirow{2}{*}{ Sensors } & \multirow{2}{*}{ Part of body } & \multicolumn{3}{|c|}{ Envelope amplitude } \\
\hline & & Before flight & After flight & increase rate [\%] \\
\hline $\begin{array}{l}\text { Reflex } \\
\text { Blue }\end{array}$ & lateral hip & $\begin{array}{c}4.98 \mathrm{~Hz} \\
3.04 \mathrm{E}-07\end{array}$ & $\begin{array}{l}5.0 \mathrm{~Hz} \\
3.38 \mathrm{E}-07\end{array}$ & 111.2 \\
\hline $\begin{array}{l}\text { Reflex } \\
\text { Green }\end{array}$ & lateral hip & $\begin{array}{c}4.9 \mathrm{~Hz} \\
1.25 \mathrm{E}-06\end{array}$ & $\begin{array}{l}5.0 \mathrm{~Hz} \\
2.84 \mathrm{E}-06\end{array}$ & 227.2 \\
\hline
\end{tabular}
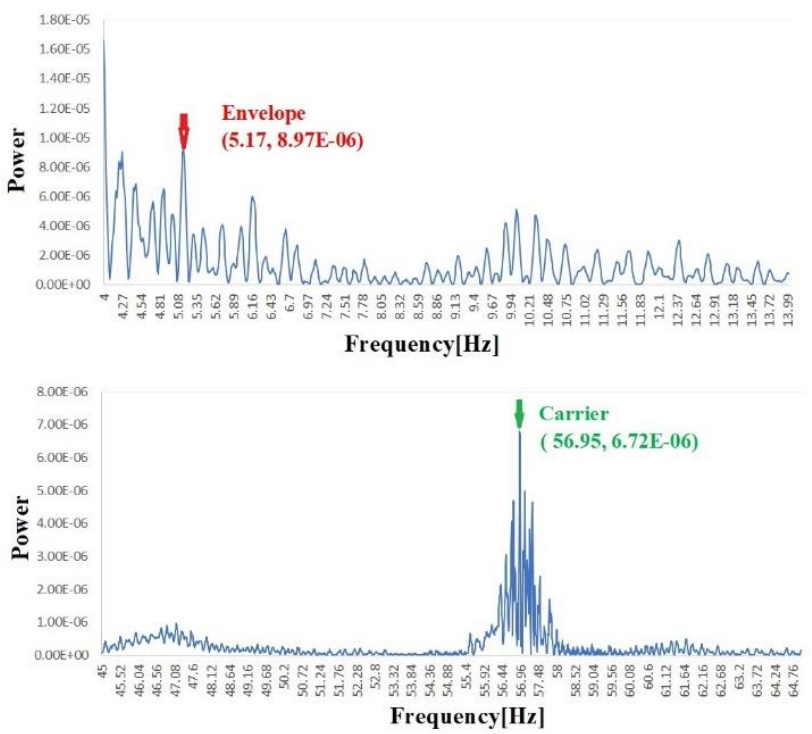

Fig. 13. Power spectrum analysis for envelope and carrier.

Based on these results, while the carrier frequency differs depending on the region (thoracic wall, pelvic, midline of back) and the action to be performed, peri odic muscle contractions are evident in all cases.

These are buried deep in the noise and found in th e frequency width removed by the low lass filter.

\section{CONSIDERATIONS}

\subsection{Why focus on the envelope?}

We derive the carrier frequency from the magnetic field (distance) between the thoracic walls, periodic noise found in the ECG, the angular velocity of the pelvic/thoracic wall, the periodic noise associated with air sac pressure, and optical waves reflected from the pelvic skin, all of which can be explained as originating from muscle vibrations. This low level of muscular tension is considered unique to birds, which lack diaphragms, and is deemed essential to maintaining constant air sac pressure. Current speculation is that such control systems are present in birds and dinosaurs, but not in mammals.

The number of envelopes is considered to represent a number of stimuli from the sympathetic nerve. Assuming that fibers from the sympathetic nerve are branched and spatially Gaussian (Figure 14) while the contraction of the muscle fibers is a trigonometric function, the observed signal is a convolution signal of both. The shape is similar to the noise and optical reflection contained in the ECG. This convolution can be simulated with a relatively simple program (Annex 1) in MALBAT.
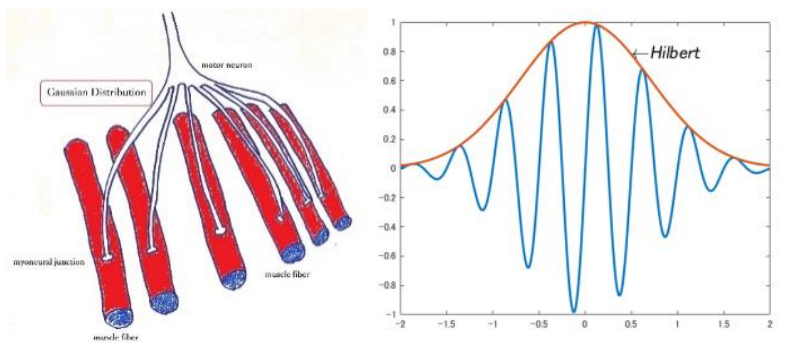

Fig. 14. The sympathetic nerves branch and are distributed according to Gaussian distribution to create envelopes. The right image was simulated by the MALTAB (Annex 1).

\subsection{Screening for diseased birds}

In an experiment using chickens, a breathing pattern was obtained using magnets attached to the thoracic wall, which were synchronized with posterior thoracic air sac pressure (with a certain time difference). Also observed were periodic oscillations of sympathetic nerve-derived intercostal muscles. This approach allows 24/365 monitoring of a chicken's respiratory status and autonomic state without contact.

As shown in Figure 15, the posterior thoracic air sac pressure and changes in the magnetic field of the thoracic wall are correlated. The respiratory patterns of the chicken and periodic fasciculations of intercostal muscles are clearly recorded.

Poultry tend to be potential low-pathogenic avian influenza carriers with the potential of mutation to high pathogenicity. Nevertheless, visually identifying birds in 
poor health remains difficult. We believe detecting antigenantibody reactions from a dead bird, based on refinements of this technology, will enable proactive crisis management that screens for suspicious individuals at an early stage and isolates them from the population, an approach preferable to culling after the fact.

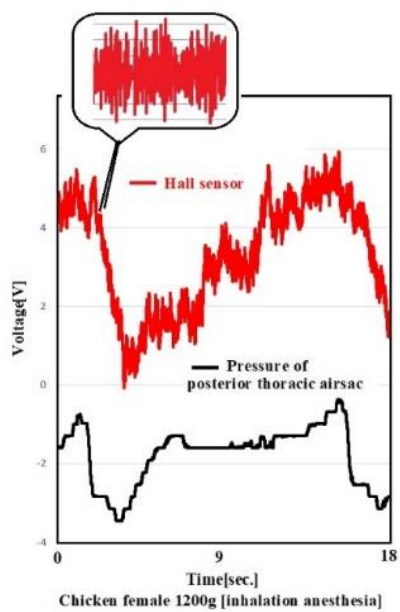

Fig. 15. Correlation between barometric pressure of posterior thoracic air sac and magnetic field with Hall sensor (hen).

Especially during forced molting (forcing starvation and measures to promote ovulation in groups that no longer lay eggs), vital signs fluctuate greatly, with many individual birds developing Newcastle disease. This study is based on the pathophysiology of avian influenza. In place of antigenantibody reactions, respiratory patterns are detected without contact using a magnet attached to the thoracic wall and a Hall sensor attached to the cage wall. This allows isolation of individual birds in the early stages of infection. Linked to a data line, this will allow local governments to monitor conditions and provide suitable guidance (Figure 16). We see this early isolation as one solution for preventing the spread of avian influenza.

There are two methods of detecting abnormalities with magnets: (a) respiratory patterns and (b) periodic fasciculations.

(a)Can the presence or absence of a notch be detected by respiratory pattern? This notch is caused especially airflow between the air sacs. This detection can be enhanced with AI (Recurrent Neural Networks). For example, with RNN, learn the normal state 1000 times, and then learn 1000 times with the low oxygen inhalation condition intentionally, in the next tern 2001, the pattern is healthy or disorder?

(b)For periodic fasciculations, which reflect the condition of sympathetic nerves, the frequency of the envelope and the strength of the carrier frequency. When the disease develops, the frequency of the envelope and the signal strength decreases.
Up to now, there is no system to monitor the vital signs of birds from the poultry house in real time. Poultry traders are owners and do not report dead bodies and sometimes sell them. This is why governments need a system to manage poultry remotely.
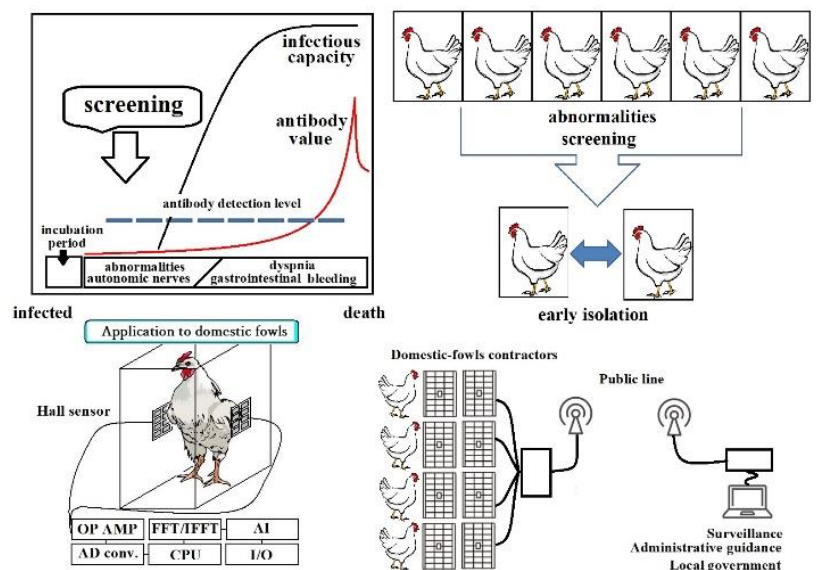

Fig. 16. Future system linked to data lines to enable early isolation and monitoring by local governments.

\section{CONCLUSION}

The following is a summary of this paper:

1. Magnets and Hall sensors sewn on the left and right thoracic walls, bipolar electrocardiograms measured between same thoracic walls, posterior thoracic air sac pressure, angular velocity sensors sewn on the back or hips, and optical reflection of LEDs (blue and green) from the skin of the hips make it possible to observe periodic vibrations (fasciculations). To date, no such analysis has been reported.

2. These fasciculations are presumed to derive from muscle, with the function of maintaining and controlling air sac pressure.

3. Since each muscle fiber is a spatial Gaussian distributed from the sympathetic nerve, it is assumed that the envelope plots a Gaussian curve.

4. Avian trunk muscles contract periodically at all times, suggesting that the sympathetic nerve controlling this is predominant.

5. The technique of sewing a magnet to the thoracic wall and measuring the strength of magnetic fields with a Hall sensor can be applied to captive animals to allow screening for the early stages of avian influenza.

\section{Acknowledgements}

This study was undertaken from 2012 to 2015 after appropriate review and approval by the institutional animal ethics committee at Tokai University. The research was funded by grants-in-aid for scientific research (Grants 
21241042 and 23651169) from the Ministry of Education, Culture, Sports, Science and Technology. For their contributions to the work described in this paper, we are deeply indebted to all those at Tokai University School of Medicine, in particular Prof. Ichiro Kuwahira, Dr. Kaoru Nakada, Prof. Yoshiya Muraki, Prof. Toshihiko Kitano, Dr. Nadia El Borai, Ms. Noriko Numata, Ms. Miyoshi Tanaka, Ms. Katsuko Naito, Ms. Yoshiko Ito and Ms. Hiroko Ichimura who were helpful at all times and provided essential assistance. We would like to also thank Prof. Kiyoshi Kurokawa of the National Graduate Institute for Policy Studies.

The data management program with the Excel VBA was supported by Mr. Hiroshi Juzoji; EFL Inc. in Takaoka City. The data logger system was designed and provided by Mr. Takahiro Kawai; limited company Rohrm Riken in Kosei City. All pheasants used in the experiments were purchased from Japan Pheasant Breeding Center in Hitachi City.

Annex 1. Program code for the simulation.

the Gaussian convolution and its Envelope

$\%$ Open source code of MATLAB for readers of $\mathrm{J}$ of

Multimedia Information System

\% Isao Nakajima, Tokai University, Nov. 30, 2019

Fs $=100 ; \%$ sampling frequency

$\mathrm{x}=-2: 1 / \mathrm{Fs}: 2 ; \%$ vector of time

$\mathrm{y}=\cos \left(4^{*} \mathrm{pi}^{*} \mathrm{x}-(\mathrm{pi} / 2)\right){ }^{*} \exp \left(-\mathrm{x} .^{\wedge} 2\right) ; \%$ Gaussian

distribution

$\mathrm{z}=\operatorname{hilbert}(\mathrm{y}) ; \%$ Hilbert

$\mathrm{w}=\operatorname{abs}(\mathrm{z})$;

plot(x,y,x,w,'LineWidth',2)

text(0.495, 0.8,''leftarrowlit\{ Hilbert\}','FontSize', 16)

filename='E: Hilbert.csv'; $\%$ save the envelope

$\mathrm{str}=[\mathrm{x} ; \mathrm{w} ;]$

csvwrite(filename,str)

type('C:Hilbert.csv')

\section{REFERENCES}

[1] OIE, "Highly pathogenic avian influenza"3 May 2018;https://www.oie.int/doc/ged/D9311.PDF/.

[2] WHO, "Updated unified nomenclature system for the highly pathogenic H5N1 avian influenza viruses" 3 May 2018;http://www.who.int/influenza/gisrs_laboratory/h5 n1_nomenclature/en/.

[3] WHO "Cumulative number of confirmed human cases of avian influenza $\mathrm{A}(\mathrm{H} 5 \mathrm{~N} 1)$ reported to WHO"3 May,2018;http://www.who.int/influenza/human_anima 1_interface/H5N1_cumulative_table_archives/en/.

[4] Shinya K, Ebina M, Yamada S, et al., "Avian flu: influenza virus receptors in the human airway," Nature
440, pp. 435-436, 2006.

[5] Nicholls J. M, Bourne A. J, Chen H et al., "Sialic acid receptor detection in the human respiratory tract: evidence for widespread distribution of potential binding sites for human and avian influenza viruses," Respiratory Research, vol.8, no.73, 2007.

[6] ITU-D RGQ 14-2/2/079-E, Avian Influenza and expected $t$ elecommunication technical development.

[7] ITU-D RGQ 14-2/2/069-E, Liaison Statement on the role of $\mathrm{t}$ elecommunication/ICT to be used for an integrated ICT network to monitor the avian influenza.

[8] ITU-D RGQ 14-2/2/070-E, Note to the Director of BDT, Proposal for an integrated ICT network to monitor the avian influenza.

[9] Working Parties 7B and 7C, Doc 7B/TEMP/387C/TEMP/22, ITU-D RGQ 14-2/2/071-E, Liaison Statement, Reply to a liaison statement from ITU-D Study group 2 regarding an ITU-D Study Group 2 Question.

[10] I. Nakajima, T. Kitano, M. Katayama, L. Androuch $\mathrm{k} 0$, “ Expected Communications Technology to Track Avian Influenza and Related the Statement of Appeal by ITU-D SG2 Q14", Int. J. eHealth \& Medical Communications vol.2, no.4, pp.20-37, 2010.

[11] I. Nakajima, "Telecommunications for Disaster and Pandemics" www.ituaj.jp/wpcontent/uploads/2013/04/nb25-2 web-10 tokai.pdf

[12] K. Nakada, I. Nakajima, J. Hata, M. Ta."Study on Vibration Energy Harvesting with Small Coil for Embedded Avian Multimedia Application," JMIS, vol. 5, no. 1.pp. 47-52, 2018.

[13] K. Nakada, I. Nakajima, J. Hata, M. Ta."Packet Transceiver on $2.4 \mathrm{GHz}$ for Whooper Swan", JMIS, vol. 5, no. 2. pp. 91-98, 2018.

[14] K. Nakada, J. Hata. "Development and physiological assessments of multimedia avian esophageal catheter system," JMIS, vol. 5, No. 2. pp. 121-130, 2018.

[15] I. Nakajima, H. Juzoji,K.Ozaki, N. Nakamura. "Communications Protocol Used in the Wireless Token Rings for Bird-to-Bird," JMIS, vol. 5, No. 3. pp. 163170, 2018.

[16] J. Maina, "The Lung-Air Sac System of Birds", Springer Berlin Heidelberg New York, 2005.

[17] P. Jewett, J. Sommer, E. Johnson, "Cardiac muscle. Its ultrastructure in the finch and hummingbird with special reference to the sarcoplasmic reticulum," J. Cell Biol., vol. 49, No. 50. 1971.

[18] P. Jewett, S. Leonard, J. Sommer. "Chicken cardiac muscle. Its elusive extended junctional sarcoplasmic reticulum and sarcoplasmic reticulum fenestrations," $J$. Cell Biol., vol. 56, no. 595. 1973.

[19] R. Harrison, "Avian Medicine: Principles and Applications," Chapter 27, Zoological Education Network, July 1994. ISBN-13: 978-0963699602.

[20] muRata, "Angular Rate Sensor ENC-03R," https://www.elecrow.com/download/ENC-03.pdf.

[21] T. Liu, Y. Inoue, K. Shibata, X.Tang, "A Wearable Inertial Sensor System for Human Motion Analysis," 
IEEE Sensors Journal, vol.16, no. 22, pp. 7821-7834, Nov. 2016.

[22] T. Fitzgerald, E. Rhee, D. Brooks, J. Triedman. "Estimation of cardiac conduction velocities using small data sets," in Proceedings of the Computers in Cardiology 2001, Sep. 2001.

[23] M. Tadi, E. Lehtonen, M. Pankäälä, A. Saraste, T. Vasankari, M. Terás, "Gyrocardiography: A new noninvasive approach in the study of mechanical motions of the heart. Concept, method and initial observations." in Proceedings of IEEE 38th Annual International Conference of Engineering in Medicine and Biology Society (EMBC), Aug. 2016.

[24] Task Force of the Europian Society of Cardiology and the North American Society of Pacing and Electrophysiology, "Heart rate variability", European Heart Journal, vol.37, pp. 354-381, 1996.

[25] F. Toyoda, T. Morita, A. Miyazaki, T. Mitsuiye, "ECG and Autonomic Nerves in Chicks", Jpn, Poult. Sci., vol. 36, pp. 260-268, 1999.

[26] D. Lakens, Using a Smartphone to Measure Heart Rate Changes during Relived Happiness and Anger," IEEE Trans. on Affective Computing, vol. 4, no. 2, pp. 238241, Apr. 2013, DOI: 1109/T-AFFC.2013.3.

[27] A. Biewener, "Muscle function in avian flight: achieving power and control," Philos Trans $R$ Soc Lond B Biol Sci., vol. 366, no. 1570, pp. 1496-1506, 2011.

[28] "Basic Description of the Argos System," Feb. 2018; www.webpages.uidaho.edu/wlf314/labs/Argos System Description.pdf.

[29] North Star, "Tracking Birds," www.northstarst.com/tracking-birds, Mar. 2018.

[30] O. Roy, J. Hart, "Transmitter for Telemetry of Biological Data from Birds in Flight", IEEE Transactions on Bio-medical Electronics, vol. 10, no. 3, pp. 114-116, July 1963.

[31] E. Morishita, K. Itao, K. Sasaki, H. Higuchi, "Movements of Crows in Urban Areas Based on PHS Tracking,"

www.airies.or.jp/attach.php/.../save/0/0/07 2-09.pdf, Feb. 2018.

[32] Y. Li, Y. Yin, "Bird Objects Detection and Tracking on the Wild Field Circumstance," in Proceedings of IEEE Computer Society WRI World Congress on Computer Science and Information Engineering, DOI: 10.1109/CSIE.2009.287, 2009.

[33] D. Brown, "Birds Fly More Than 7,000 Miles Nonstop, Study Shows," Washington Post, October 22, 2008.
University 1988, and the Medical Doctor (M.D.) from Tokai University School of Medicine 1980. He has been aiming to send huge multimedia data from moving ambulance via communications satellite to assist patient's critical condition.

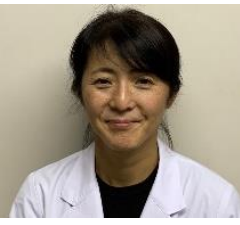

Sachie Tanaka has operated and supported animal experiments in the Dept. of Laboratory Animal Science, Support Center for Medical Research and Education, Tokai University as a senior technician. She graduated from Tokai University School of Agriculture in 1994 and got a Bachelor of Agriculture.

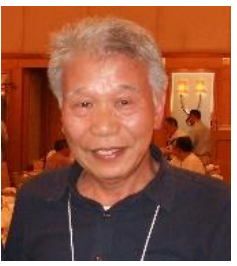

Kokuryo Mitsuhashi worked at JVC for 17 years and engaged in research and development of electronic devices. He has developed and designed high-frequency analog circuits that generate plasma at semiconductor manufacturing equipment manufacturers and taught these technologies at the University of Tokyo and major semiconductor manufacturing equipment manufacturers.

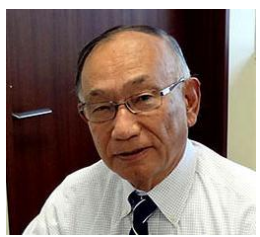

Jun-ichi Hata has received his MD degree from Keio University School of Medicine, Tokyo, in 1966. In 1971, he received a PhD degree in Keio University Post Graduate School of Medicine. Since then, he has been mainly involved in molecular pathology for pediatric tumors and embryonal pathology. He was appointed to a professor of pathology, Keio University School of Medicine in 1990. In 2013, he has been appointed as research director of Central Institute for Experimental Animals from 2013.

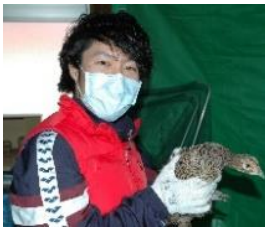

Tomo Nakajima is an infectious disease specialist and currently works at Okinawa Prefectural Yaeyama Hospital. He graduated from Tokai University School of Medicine (M.D.) in 2014 and has been a resident at Okinawa Prefectural Chubu Hospital since 2016. He is also interested in hematology and related fields. His hobby is photography.

\section{Authors}

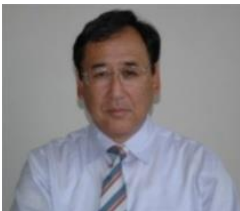

Isao Nakajima worked at Tokai University School of Medicine as Professor of Nakajima Laboratory, Department of Emergency Medicine and Critical Care. He got the Doctor of Applied Informatics (Ph.D.), Graduate School of Applied Informatics University of Hyogo 2009, and the Doctor of Medicine (Ph.D.), Post Graduate School of Medical Science Tokai 
Detecting of Periodic Fasciculations of Avian Muscles Using Magnetic and Other Multimedia Devices 